\title{
Bijective evaluation of the connection coefficients of the double coset algebra
}

\author{
Alejandro H. Morales ${ }^{1}$ and Ekaterina A. Vassilieva ${ }^{2}$ \\ ${ }^{1}$ Department of Mathematics, Massachusetts Institute of Technology, Cambridge, MA, USA \\ ${ }^{2}$ Laboratoire d'Informatique de l'Ecole Polytechnique, Palaiseau, France
}

\begin{abstract}
This paper is devoted to the evaluation of the generating series of the connection coefficients of the double cosets of the hyperoctahedral group. Hanlon, Stanley, Stembridge (1992) showed that this series, indexed by a partition $\nu$, gives the spectral distribution of some random matrices that are of interest in random matrix theory. We provide an explicit evaluation of this series when $\nu=(n)$ in terms of monomial symmetric functions. Our development relies on an interpretation of the connection coefficients in terms of locally orientable hypermaps and a new bijective construction between partitioned locally orientable hypermaps and some permuted forests.

Résumé. Cet article est dédié à l'évaluation des séries génératrices des coefficients de connexion des classes doubles (cosets) du groupe hyperoctaédral. Hanlon, Stanley, Stembridge (1992) ont montré que ces séries indexées par une partition $\nu$ donnent la distribution spectrale de certaines matrices aléatoires jouant un rôle important dans la théorie des matrices aléatoires. Nous fournissons une évaluation explicite de ces séries dans le cas $\nu=(n)$ en termes de monômes symétriques. Notre développement est fondé sur une interprétation des coefficients de connexion en termes d'hypercartes localement orientables et sur une nouvelle bijection entre les hypercartes localement orientables partitionnées et certaines forêts permutées.
\end{abstract}

Keywords: double coset algebra, connection coefficients, locally orientable hypermaps, forests

\section{Introduction}

In what follows, we denote by $\lambda=\left(\lambda_{1}, \lambda_{2}, \ldots, \lambda_{k}\right)_{\geq} \vdash n$ an integer partition of $n$ and $\ell(\lambda)=k$ the number of parts of $\lambda$. If $n_{i}(\lambda)$ is the number of parts of $\lambda$ that are equal to $i$ (by convention $n_{0}(\lambda)=0$ ), then we write $\lambda$ as $1^{n_{1}(\lambda)} 2^{n_{2}(\lambda)} \ldots$ and let $A u t_{\lambda}=\prod_{i} n_{i}(\lambda)$ !. Also, if $\lambda \vdash n$, let $\lambda \lambda$ and $2 \lambda$ be the partitions of $2 n\left(\lambda_{1}, \lambda_{1}, \lambda_{2}, \lambda_{2}, \ldots\right)$ and $\left(2 \lambda_{1}, 2 \lambda_{2}, \ldots\right)$ respectively. Let $[m]=\{1, \ldots, m\}$ and $S_{m}$ be the symmetric group on $m$ elements, e.g. on $[m]$, and let $\mathcal{C}_{\lambda}$ be the conjugacy class in $S_{m}$ of permutations $w$ with cycle type type $(w)=\lambda \vdash m$.

We look at perfect pairings of the set $[n] \cup[\widehat{n}]=\{1, \ldots n, \widehat{1}, \ldots, \widehat{n}\}$ which we view as fixed point free involutions in $S_{2 n}([n] \cup[\widehat{n}])$. Note that for $f, g \in S_{2 n}$, the disjoint cycles of the product $f \circ g$ have repeated lengths i.e. $f \circ g \in \mathcal{C}_{\lambda \lambda}$. Also, for $w \in S_{2 n}$, let $f_{w}$ be the pairing $\left(w^{-1}(1), w^{-1}(\hat{1})\right) \cdots\left(w^{-1}(n), w^{-1}(\hat{n})\right)$.

Let $B_{n}$ be the hyperoctahedral group which we view as the centralizer in $S_{2 n}$ of the involution $f_{\star}=$ $(1 \widehat{1})(2 \widehat{2}) \cdots(n \widehat{n})$. Then $\left|B_{n}\right|=2^{n} n$ !, and it is well known that the double cosets of $B_{n}$ in $S_{2 n}$ are indexed by partitions $\nu$ of $n$, and consist of permutations $w \in S_{2 n}$ such that the cycle type of $f_{\star} \circ f_{w}$ is $\nu \nu[8$, Ch. 
VII.2]. If we denote such double coset by $K_{\nu}$ and pick from it a fixed element $w_{\nu}$, then let $b_{\lambda, \mu}^{\nu}$ be the number of ordered factorizations $u_{1} \cdot u_{2}$ of $w_{\nu}$ where $u_{1} \in K_{\lambda}$ and $u_{2} \in K_{\mu}$. We provide a combinatorial formula for $b_{\lambda, \mu}^{\nu}$ when $\nu=(n)=n$ (say $\left.w_{(n)}=(123 \ldots n)(\widehat{n} n \widehat{-1} 1 \hat{n-2} \ldots \widehat{1})\right)$ by interpreting these factorizations as locally orientable (partitioned) unicellular hypermaps.

Theorem 1.1 Let $b_{\lambda, \mu}^{n}$ be the number of ordered factorizations $u_{1} \cdot u_{2}$ of $w_{(n)}$ where $u_{1} \in K_{\lambda}$ and $u_{2} \in K_{\mu}$. If $p_{\lambda}(\mathbf{x})$ and $m_{\lambda}(\mathbf{x})$ are the power and monomial symmetric functions then

$$
\begin{aligned}
\frac{1}{2^{n} n !} \sum_{\lambda, \mu \vdash n} b_{\lambda, \mu}^{n} p_{\lambda}(\mathbf{x}) p_{\mu}(\mathbf{y})= & \\
& \sum_{\lambda, \mu \vdash n} A u t_{\lambda} A u t_{\mu} m_{\lambda}(\mathbf{x}) m_{\mu}(\mathbf{y}) \sum_{\mathbf{A} \in M_{\lambda, \mu}} \frac{\mathcal{N}(\mathbf{A})}{\mathbf{A} !} \frac{(n-q-2 r) !(n-p-2 r) !}{(n+1-p-q-2 r) !} \\
& \frac{p^{\prime} ! q^{\prime} !\left(r-p^{\prime}\right) !\left(r-q^{\prime}\right) !}{2^{2 r-p^{\prime}-q^{\prime}}} \prod_{i, j, k}\left(\begin{array}{c}
i-1 \\
j, k, j+k
\end{array}\right)^{(P+Q)_{i, j, k}}\left(\begin{array}{c}
i-1 \\
j, k, j+k-1
\end{array}\right)
\end{aligned}
$$

Where, $M_{\lambda, \mu}$ is the set of 4-tuples $\mathbf{A}=\left(P, P^{\prime}, Q, Q^{\prime}\right)$ of tridimensional arrays of non negative integers with $p=|P|=\sum_{i, j, k \geq 0} P_{i j k} \neq 0, p^{\prime}=\ell(\lambda)-p=\left|P^{\prime}\right|, q=|Q|, q^{\prime}=\ell(\mu)-q=\left|Q^{\prime}\right|$, and

$$
\begin{aligned}
& n_{i}(\lambda)=\sum_{j, k} P_{i j k}+P_{i j k}^{\prime}, \quad n_{i}(\mu)=\sum_{j, k \geq 0} Q_{i j k}+Q_{i j k}^{\prime}, \\
& r=\sum_{i, j, k}(j+k)\left(P_{i j k}+P_{i j k}^{\prime}\right), \quad r=\sum_{i, j, k}(j+k)\left(Q_{i j k}+Q_{i j k}^{\prime}\right), \\
& q^{\prime}=\sum_{i, j, k} j\left(P_{i j k}+P_{i j k}^{\prime}\right), \quad p^{\prime}=\sum_{i, j, k} j\left(Q_{i j k}+Q_{i j k}^{\prime}\right) \text {. }
\end{aligned}
$$

where $\mathbf{A} !=\prod_{i, j, k} P_{i j k} ! P_{i j k}^{\prime} ! Q_{i j k} ! Q_{i j k}^{\prime} !$. And $\mathcal{N}(\mathbf{A})=\sum_{t, u, v} t P_{t u v}$ if $q^{\prime}=0$, otherwise if $q^{\prime} \neq 0$ then

$$
\begin{aligned}
& \mathcal{N}(\mathbf{A})= \\
& \frac{1}{q^{\prime}} \sum_{t-2 u-2 v>0} \frac{t P_{t u v}}{t-2 u-2 v}\left[(t-2 u-2 v)\left(\frac{\delta_{p^{\prime} \neq 0}}{p^{\prime}} \sum_{i, j, k} j Q \sum_{i, j, k} j P^{\prime}+\frac{\sum_{i, j, k}(i-1-2 j-2 k) Q \sum_{i, j, k} j P}{n-q-2 r}\right)\right. \\
& \left.\quad+u\left(\frac{\delta_{p^{\prime} \neq 0}}{p^{\prime}} \sum_{i, j, k}(i-2 j-2 k) P^{\prime} \sum_{i, j, k} j Q^{\prime}+\sum_{i, j, k}(i-2 j-2 k) Q^{\prime} \frac{1+\sum_{i, j, k}(i-1-2 j-2 k) P}{n-q-2 r}\right)\right] .
\end{aligned}
$$

Remark 1.2 For some limit values of the 4-tuple $\mathbf{A}$, the summand definition in $\sum_{\mathbf{A} \in M_{\lambda, \mu}} \cdots$ on the RHS of Equation (1) is slightly different as detailed in Appendix 6 of the paper.

\subsection{Background on connection coefficients $b_{\lambda, \mu}^{\nu}$}

By abuse of notation, let the double coset $K_{\nu}$ also represent the sum of its elements in the group algebra $\mathbb{C} S_{2 n}$. Then $K_{\nu}$ form a basis of a commutative subalgebra of $\mathbb{C} S_{2 n}$ (the Hecke algebra of the Gelfand pair $\left.\left(S_{2 n}, B_{n}\right)\right)$ and one can check that $K_{\lambda} \cdot K_{\mu}=\sum_{\nu} b_{\lambda, \mu}^{\nu} K_{\nu}$. Thus, $\left\{b_{\lambda, \mu}^{\nu}\right\}$ are the connection coefficients of this double coset algebra. We use $Z_{\lambda}(\mathbf{x})$ to denote the zonal polynomial indexed by $\lambda$ which can be viewed as an analogue of the Schur function $s_{\lambda}$ (for more information on these polynomials see [8, Ch. VII]). In terms of $p_{\mu}: s_{\lambda}(\mathbf{x})=\sum_{\mu} z_{\mu}^{-1} \chi_{\mu}^{\lambda} p_{\mu}(\mathbf{x})$ where $z_{\lambda}=A u t_{\lambda} \prod_{i} i^{n_{i}(\lambda)}, \chi_{\mu}^{\lambda}$ are the irreducible characters of the symmetric group; and $Z_{\lambda}(\mathbf{x})=\frac{1}{\left|B_{n}\right|} \sum_{\mu \vdash n} \varphi^{\lambda}(\mu) p_{\mu}(\mathbf{x})$ where $\varphi^{\lambda}(\mu)=\sum_{w \in K_{\mu}} \chi_{\text {type }(w)}^{2 \lambda}$. 
In [5. Lemma 3.3] a formula for the connection coefficients in terms of $\chi_{\mu}^{\lambda}$ was given:

$$
b_{\lambda, \mu}^{\nu}=\frac{1}{\left|K_{\nu}\right|} \sum_{\beta \vdash n} \frac{1}{H_{2 \nu}} \varphi^{\beta}(\nu) \varphi^{\beta}(\lambda) \varphi^{\beta}(\mu),
$$

where $\left|K_{\nu}\right|=\left|B_{n}\right|\left|\mathcal{C}_{\nu}\right| 2^{n-\ell(\nu)}$ [2, Lemma 2.1], and $H_{2 \lambda}$ is the product of all the hook-lengths of the partition $2 \lambda$.

Let $\Psi^{\nu}(\mathbf{x}, \mathbf{y})=\frac{1}{\left|B_{n}\right|} \sum_{\lambda, \mu} b_{\lambda, \mu}^{\nu} p_{\lambda}(\mathbf{x}) p_{\mu}(\mathbf{y})$. So $\Psi^{(n)}$ is the LHS of (1). Equation (2) immediately implies that $\Psi^{\nu}(\mathbf{x}, \mathbf{y})=\frac{1}{\left|K_{\nu}\right|} \sum_{\lambda \vdash n} \frac{\left|B_{n}\right|}{H_{2 \lambda}} \varphi^{\lambda}(\nu) Z_{\lambda}(\mathbf{x}) Z_{\mu}(\mathbf{y})$. Moreover, if for an $n \times n$ matrix $X$ we say that $p_{k}(X)=\operatorname{trace}\left(X^{k}\right)$, then in [5, Thm. 3.5] it was shown that $\Psi^{\nu}$ is also the expectation of $p_{\nu}\left(X U Y U^{T}\right)$ over $U$, where $U$ are $n \times n$ matrices whose entries are independent standard normal random real variables and $X, Y$ are arbitrary but fixed real symmetric matrices.

\section{Combinatorial formulation}

\subsection{Unicellular locally orientable hypermaps}

From a topological point of view, a locally orientable hypermap of $\mathrm{n}$ edges can be defined as a connected bipartite graph with black and white vertices. Each edge is composed of two half edges both connecting the two incident vertices. This graph is embedded in a locally orientable surface such that if we cut the graph from the surface, the remaining part consists of connected components called faces or cells, each homeomorphic to an open disk. The map can be represented as a ribbon graph on the plane keeping the incidence order of the edges around each vertex. In such a representation, two half edges can be parallel or cross in the middle. A crossing (or a twist) of two half edges indicates a change of orientation in the map and that the map is embedded in a non orientable surface (projective plane, Klein bottle,...). We say a hypermap is rooted if it has a distinguished half edge. In [2], it was shown that rooted hypermaps admit a natural formal description involving triples of perfect pairings $\left(f_{1}, f_{2}, f_{3}\right)$ on the set of half edges where:

- $f_{3}$ associates half edges of the same edge,

- $f_{1}$ associates immediately successive (i.e. with no other half edges in between) half edges moving around the white vertices, and

- $f_{2}$ associates immediately successive half edges moving around the black vertices.

Formally we label each half edge with an element in $[n] \cup[\widehat{n}]=\{1, \ldots, n, \widehat{1}, \ldots, \widehat{n}\}$, labelling the rooted half edge by 1 . We then define $\left(f_{1}, f_{2}, f_{3}\right)$ as perfect pairings on this set. Combining the three pairings gives the fundamental characteristics of the hypermap since:

- The cycles of $f_{3} \circ f_{1}$ give the succession of edges around the white vertices. If $f_{3} \circ f_{1} \in \mathcal{C}_{\lambda \lambda}$ then the degree distribution of the white vertices is $\lambda$ (counting only once each pair of half edges belonging to the same edge),

- The cycles of $f_{3} \circ f_{2}$ give the succession of edges around the black vertices. If $f_{3} \circ f_{2} \in \mathcal{C}_{\mu \mu}$ then the degree distribution of the black vertices is $\mu$ (counting only once each pair of half edges belonging to the same edge),

- The cycles of $f_{1} \circ f_{2}$ encode the faces of the map. If $f_{1} \circ f_{2} \in \mathcal{C}_{\nu \nu}$ then the degree distribution of the faces is $\nu$

In what follows, we consider the number $L_{\lambda, \mu}^{n}$ of rooted unicellular, or one-face, locally orientable hypermaps with face distribution $\nu=(n)=n^{1}$, white vertex distribution $\lambda$, and black vertex distribution $\mu$. 
Let $f_{1}$ be the pairing $(1 \widehat{n})(2 \widehat{1})(3 \widehat{2}) \ldots(n n \widehat{-} 1)$ and $f_{2}=f_{\star}=(1 \widehat{1})(2 \widehat{2}) \ldots(n \widehat{n})$. We have $f_{1} \circ f_{2}=$ $(123 \ldots n)(\widehat{n} n \widehat{-1} n \widehat{-2} \ldots \widehat{1}) \in \mathcal{C}_{(n)(n)}$. Then by the above description, one can show that

$$
L_{\lambda, \mu}^{n}=\mid\left\{f_{3} \text { pairings in } S_{2 n}([n] \cup[\widehat{n}]) \mid f_{3} \circ f_{1} \in \mathcal{C}_{\lambda \lambda}, f_{3} \circ f_{2} \in \mathcal{C}_{\mu \mu}\right\} \mid .
$$

Moreover, the following relation between $L_{\lambda, \mu}^{n}$ and $b_{\lambda, \mu}^{n}$ holds [2, Cor 2.3]

$$
L_{\lambda, \mu}^{n}=\frac{1}{2^{n} n !} b_{\lambda, \mu}^{n}
$$

Thus we can encode the connection coefficients as numbers of locally orientable hypermaps.

Example 2.1 Figure 1 depicts a locally orientable unicellular hypermap in $L_{\lambda, \mu}^{n}$ with $\lambda=1^{1} 2^{2} 3^{1} 4^{1}$ and $\mu=3^{1} 4^{1} 5^{1}$ (at this stage we disregard the geometric shapes around the vertices).

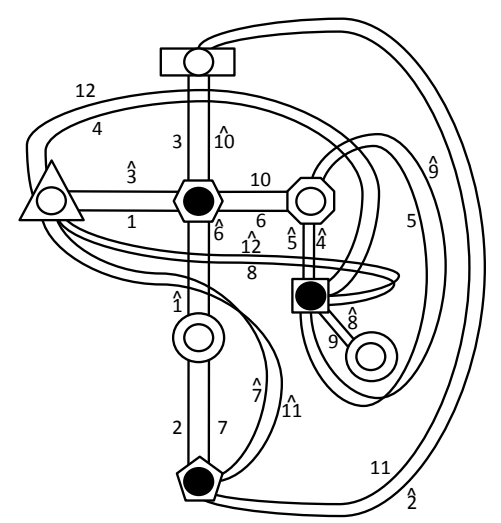

Fig. 1: A unicellular locally orientable hypermap

\subsection{Partitioned locally orientable hypermaps}

Next, we consider locally orientable hypermaps where we partition the white vertices (black resp.). In terms of the pairings, this means we "color" the cycles of $f_{3} \circ f_{1}\left(f_{3} \circ f_{2}\right.$ resp. $)$ allowing repeated colors but imposing that the two cycles corresponding to each white (black resp.) vertex have the same color. The following definition in terms of set partitions of $[n] \cup[\widehat{n}]$ makes this more precise.

Definition 2.2 (Locally orientable partitioned hypermaps) We consider the set $\mathcal{L P}_{\lambda, \mu}^{n}$ of triples of the form $\left(f_{3}, \pi_{1}, \pi_{2}\right)$ where $f_{3}$ is a pairing on $[n] \cup[\widehat{n}], \pi_{1}$ and $\pi_{2}$ are sets partitions on $[n] \cup[\widehat{n}]$ with blocks of even size and of respective types $2 \lambda$ and $2 \mu$ (or half types $\lambda$ and $\mu$ ) with the constraint that $\pi_{i}(i=1,2)$ is stable by $f_{i}$ and $f_{3}$. Any such triple is called a locally orientable partitioned hypermap of type $(\lambda, \mu)$. In addition, let $L P_{\lambda, \mu}^{n}=\left|\mathcal{L} \mathcal{P}_{\lambda, \mu}^{n}\right|$.

Remark 2.3 The analogous notion of partitioned or colored map is common in the study of orientable maps. e.g. see [7], [4] for maps. Recently Bernardi in [1] extended the approach in [7] to find a bijection between locally orientable partitioned maps and orientable partitioned maps with a distinguished planar submap. As far as we know [1] Sect. 7] this technique does not extend to locally orientable hypermaps. 
Lemma 2.4 The number of hat numbers in a block is equal to the number of non hat numbers

Proof: If a non hat number $i$ belongs to block $\pi_{1}^{k}$ then $f_{1}(i)=i \widehat{-1}$ also belongs to $\pi_{1}^{k}$. The same argument applies to blocks of $\pi_{2}$ with $f_{2}(i)=\widehat{i}$.

Example 2.5 As an example, the locally orientable hypermap on Figure 1 is partitioned by the blocks:

$$
\begin{aligned}
& \pi_{1}=\{\{\widehat{12}, 1, \widehat{3}, 4, \widehat{7}, 8, \widehat{11}, 12\} ;\{\widehat{1}, 2, \widehat{6}, 7, \widehat{8}, 9\} ;\{\widehat{2}, 3, \widehat{10}, 11\} ;\{\widehat{4}, 5, \widehat{5}, 6, \widehat{9}, 10\}\} \\
& \pi_{2}=\{\{1, \widehat{1}, 3, \widehat{3}, 6, \widehat{6}, 10, \widehat{10}\} ;\{2, \widehat{2}, 7, \widehat{7}, 11, \widehat{11}\} ;\{4, \widehat{4}, 5, \widehat{5}, 8, \widehat{8}, 9, \widehat{9}, 12, \widehat{12}\}\}
\end{aligned}
$$

(blocks are depicted by the geometric shapes around the vertices, all the vertices belonging to a block have the same shape).

Let $\bar{R}_{\lambda, \mu}$ be the number of unordered partitions $\pi=\left\{\pi^{1}, \ldots, \pi^{p}\right\}$ of the set $[\ell(\lambda)]$ such that $\mu_{j}=$ $\sum_{i \in \pi^{j}} \lambda_{i}$ for $1 \leq j \leq \ell(\mu)$. Then for the monomial and power symmetric functions, $m_{\lambda}$ and $p_{\lambda}$, we have: $p_{\lambda}=\sum_{\mu \succeq \lambda} A u t_{\mu} \bar{R}_{\lambda, \mu} m_{\mu}$. We use this to obtain a relation between $L_{\lambda, \mu}^{n}$ and $L P_{\lambda, \mu}^{n}$

Proposition 2.1 For partitions $\rho, \epsilon \vdash n$ we have $L P_{\nu, \rho}^{n}=\sum_{\lambda, \mu} \bar{R}_{\lambda \nu} \bar{R}_{\mu \rho} L_{\lambda, \mu}^{n}$, where $\lambda$ and $\mu$ are refinements of $\nu$ and $\rho$ respectively.

Proof: Let $\left(f_{3}, \pi_{1}, \pi_{2}\right) \in \mathcal{L P}_{\nu, \rho}^{n}$. If $f_{3} \circ f_{1} \in \mathcal{C}_{\lambda \lambda}$ and $f_{3} \circ f_{2} \in \mathcal{C}_{\mu \mu}$ then by definition of the set partitions we have that $\lambda$ and $\mu$ are refinements of type $\left(\pi_{1}\right)=\nu$ and type $\left(\pi_{2}\right)=\rho$ respectively. Thus, we can classify the elements of $\mathcal{L} \mathcal{P}_{\nu, \rho}^{n}$ by the cycle types of $f_{3} \circ f_{1}$ and $f_{3} \circ f_{2}$. i.e. $\mathcal{L} \mathcal{P}_{\nu, \rho}^{n}=\bigcup_{\lambda, \mu} \mathcal{L P}_{\nu, \rho}^{n}(\lambda, \mu)$ where

$$
\mathcal{L P}_{\nu, \rho}(\lambda, \mu)=\left\{\left(f_{3}, \pi_{1}, \pi_{2}\right) \in \mathcal{L P}_{\nu, \rho}^{n} \mid\left(f_{3} \circ f_{1}, f_{3} \circ f_{2}\right) \in \mathcal{C}_{\lambda \lambda} \times \mathcal{C}_{\mu \mu}\right\} .
$$

If $L P_{\mu \rho}^{n}(\lambda, \mu)=\left|\mathcal{L} \mathcal{P}_{\mu \rho}^{n}(\lambda, \mu)\right|$ then it is easy to see that $L P_{\mu, \rho}^{n}(\lambda, \mu)=\bar{R}_{\lambda \nu} \bar{R}_{\mu \rho} L_{\lambda \mu}^{n}$.

By the change of basis equation between $p_{\lambda}$ and $m_{\lambda}$, this immediately relates the generating series $\Psi^{n}$ and the generating series for $L P_{\lambda, \mu}^{n}$ in monomial symmetric functions. i.e. $\Psi^{n}(\mathbf{x}, \mathbf{y})$ is

$$
\sum_{\lambda, \mu \vdash n} L_{\lambda, \mu}^{n} p_{\lambda}(\mathbf{x}) p_{\mu}(\mathbf{y})=\sum_{\lambda, \mu \vdash n} A u t_{\lambda} A u t_{\mu} L P_{\lambda, \mu}^{n} m_{\lambda}(\mathbf{x}) m_{\mu}(\mathbf{y}) .
$$

Definition 2.6 Let $\mathcal{L} \mathcal{P}(\mathbf{A})$ be the set, of cardinality $L P(\mathbf{A})$, of partitioned locally orientable hypermaps with $n$ edges where $\mathbf{A}=\left(P, P^{\prime}, Q, Q^{\prime}\right)$ are tridimensional arrays such that for $i, j, k \geq 0$ :

- $P_{i j k}$ (resp. $P_{i j k}^{\prime}$ ) is the number of blocks of $\pi_{1}$ of half size $i$ such that:

(i) either 1 belongs to the block or its maximum non-hat number is paired to a hat number by $f_{3}$ (resp. blocks of $\pi_{1}$ not containing 1 such that the maximum non-hat number of the block is paired to a non-hat number by $\left.f_{3}\right)$,

(ii) the block contains $j$ pairs $\left\{t, f_{3}(t)\right\}$ where $t$ is the maximum hat number of a block of $\pi_{2}$ such that $f_{3}(t)$ is also a hat number, and,

(iii) the block contains as a whole $j+k$ pairs $\left\{u, f_{3}(u)\right\}$ where both $u$ and $f_{3}(u)$ are non-hat numbers.

- $Q_{i j k}\left(\right.$ resp. $\left.Q_{i j k}^{\prime}\right)$ is the number of blocks of $\pi_{2}$ of half size $i$ such that: 
(i) the maximum hat number of the block is paired to a non-hat (resp. hat) number by $f_{3}$,

(ii) the block contains $j$ pairs $\left\{t, f_{3}(t)\right\}$ where $t$ is the maximum non-hat number of a block of $\pi_{1}$ non containing 1 and such that $f_{3}(t)$ is also a non-hat number, and

(iii) the block contains as a whole $j+k$ pairs $\left\{u, f_{3}(u)\right\}$ where both $u$ and $f_{3}(u)$ are hat numbers.

As a direct consequence, for $\operatorname{LP}(\mathbf{A})$ to be non zero $\mathbf{A}$ has to check the conditions of Theorem 1.1 Furthermore:

$$
L P_{\lambda, \mu}^{n}=\sum_{\mathbf{A} \in M_{\lambda, \mu}} L P(\mathbf{A})
$$

Example 2.7 The partitioned hypermap on Figure 1 belongs to $\mathcal{L P}(\mathbf{A})$ for $P=E_{4,1,0}+E_{3,0,1}+E_{2,0,0}$, $P^{\prime}=E_{3,0,1}, Q=E_{5,0,1}+E_{4,1,0}, Q^{\prime}=E_{3,0,1}$ where $E_{t, u, v}$, the elementary array with $E_{t, u, v}=1$ and 0 elsewhere.

One can notice that a hypermap is orientable if and only if $f_{3}(t)$ is a hat number when $t$ is a non hat number (we go through each edge of the map in both directions and there are no changes of direction during the map traversal). As a result, a hypermap in $\mathcal{L P}(\mathbf{A})$ is orientable if and only if:

- $p^{\prime}=q^{\prime}=r=0$ and

- $P_{i j k}=Q_{i j k}=0$ if $j>0$ and $/$ or $k>0$.

In this particular case, we have the following values for $\mathcal{N}(\mathbf{A})$ and $\mathbf{A}$ ! :

- $\mathcal{N}(\mathbf{A})=\sum_{i, i, k} i P_{i j k}=\sum_{t} i n_{i}(\lambda)=n$

- $\mathbf{A} !=\prod_{i} P_{i, 0,0} ! Q_{i, 0,0} !=$ Aut $_{\lambda} A u t_{\mu}$

If we denote $c_{\lambda, \mu}^{n}$ the number of such orientable maps, by Theorem 1.1. Equation 6 . Lemma 5 and Relation 4 we recover the following combinatorial result from [9, Thm. 1]:

Corollary 2.8 [9] Thm. 1]

$$
\sum_{\lambda, \mu \vdash n} c_{\lambda, \mu}^{n} p_{\lambda}(\mathbf{x}) p_{\mu}(\mathbf{y})=n \sum_{\lambda, \mu \vdash n} \frac{(n-\ell(\lambda)) !(n-\ell(\mu)) !}{(n+1-\ell(\lambda)-\ell(\mu)) !} m_{\lambda}(\mathbf{x}) m_{\mu}(\mathbf{y}) .
$$

Remark 2.9 Note that $\left\{c_{\lambda, \mu}^{n}\right\}_{\lambda, m u}$ are better known as the connection coefficients of the symmetric group which count the number of ordered factorizations $w_{1} \cdot w_{2}$ of the long cycle $(1,2, \ldots, n)$ in $S_{n}$ where $w_{1} \in \mathcal{C}_{\lambda}$ and $w_{2} \in \mathcal{C}_{\mu}$.

\subsection{Permuted forests and reformulation of the main theorem}

We show that partitioned locally orientable hypermaps admit a nice bijective interpretation in terms of some recursive forests defined as follows:

Definition 2.10 (Rooted bicolored forests of degree A) In what follows we consider the set $\mathcal{F}(\mathbf{A})$ of permuted rooted forests composed of:

- a bicolored identified ordered seed tree with a white root vertex,

- other bicolored ordered trees, called non-seed trees with either a white or a black root vertex,

- each vertex of the forest has three kind of ordered descendants : tree-edges (connecting a white and a black vertex), thorns (half edges connected to only one vertex) and loops connecting a vertex to itself. The two extremities of the loop are part of the ordered set of descendants of the incident vertex and therefore the loop can be intersected by thorns, edges and other loops as well. 
The forests in $\mathcal{F}(\mathbf{A})$ also have the following properties:

(i) the root vertices of the non-seed trees have at least one descending loop with one extremity being the rightmost descendant of the considered vertex,

(ii) the total number of thorns (resp. loops) connected to the white vertices is equal to the number of thorns (resp. loops) connected to the black ones,

(iii) there is a bijection between thorns connected to white vertices and the thorns connected to black vertices. The bijection between thorns will be encoded by assigning the same symbolic latin labels $\{a, b, c, \ldots\}$ to thorns associated by this bijection,

(iv) there is a mapping that associates to each loop incident to a white (resp. black) vertex, a black (resp. white) vertex $\mathrm{v}$ such that the number of white (resp. black) loops associated to a fixed black (resp. white) vertex $\mathrm{v}$ is equal to its number of incident loops. We will use symbolic greek labels $\{\alpha, \beta, \ldots\}$ to associate loops with vertices except for the maximal loop of a root vertex $\mathrm{r}$ of the non-seed trees. In this case, we draw an arrow (-_-_) outgoing from the root vertex $\mathrm{r}$ and incoming to the vertex associated with the loop. Arrows are non ordered, and

(v) the ascendant/descendant structure defined by the edges of the forest and the arrows defined above is a tree structure rooted in the root of the seed tree.

Finally the degree $\mathbf{A}$ of the forest is given in the following way:

(vii) $P_{i j k}$ (resp $P_{i j k}^{\prime}$ ) counts the number of non root white vertices (including the root of the seed tree) (resp. white root vertices excluding the root of the seed tree) of degree $i$, with $j$ incoming arrows and a total of $j+k$ loops.

(viii) $Q_{i j k}\left(\right.$ resp $\left.Q_{i j k}^{\prime}\right)$ counts the number of non root black vertices (resp. black root vertices) of degree $i$, with $j$ incoming arrows and total $j+k$ loops.

Example 2.11 As an example, Figure 2 depicts two permuted forests. The one on the left is of degree $\mathbf{A}=\left(P, P^{\prime}, Q, Q^{\prime}\right)$ for $P=E_{4,1,0}+E_{3,0,1}+E_{2,0,0}, P^{\prime}=E_{3,0,1}, Q=E_{5,0,1}+E_{4,1,0}$, and $Q^{\prime}=E_{3,0,1}$ while the one on the right is of degree $\mathbf{A}^{(\mathbf{2})}=\left(P^{(2)}, P^{\prime(2)}, Q^{(2)}, Q^{\prime(2)}\right)$ for $P^{(2)}=E_{7,0,3}+E_{4,1,0}$, $P^{\prime(2)}=\{0\}_{i, j, k}, Q^{(2)}=E_{7,0,2}$, and $Q^{\prime(2)}=E_{4,0,2}$.
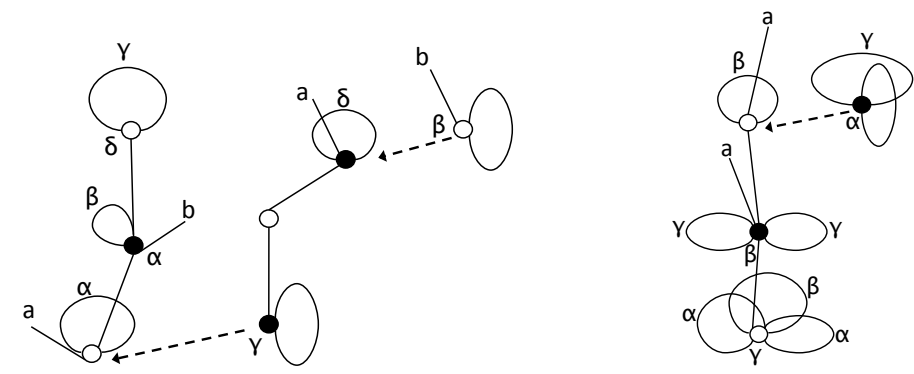

Fig. 2: Two Permuted Forests

Lemma 2.12 Using the Lagrange theorem for implicit functions, one can show that $F(\mathbf{A})$ equals

$\frac{\mathcal{N}(\mathbf{A})}{\mathbf{A} !} \frac{(n-q-2 r) !(n-p-2 r) !}{(n+1-p-q-2 r) !} \frac{p^{\prime} ! q^{\prime} !\left(r-p^{\prime}\right) !\left(r-q^{\prime}\right) !}{2^{2 r-p^{\prime}-q^{\prime}}} \prod_{i, j, k}\left(\begin{array}{c}i-1 \\ j, k, j+k\end{array}\right)^{(P+Q)_{i j k}}\left(\begin{array}{c}i-1 \\ j, k, j+k-1\end{array}\right)^{\left(P^{\prime}+Q^{\prime}\right)_{i j k}}$. 
Reformulation of the main theorem

In order to show Theorem 1.1 the next sections are dedicated to the proof of the following stronger result:

Theorem 2.13 There is a bijection $\Theta_{\mathbf{A}}: \mathcal{L P}(\mathbf{A}) \rightarrow \mathcal{F}(\mathbf{A})$ and so $L P(\mathbf{A})=F(\mathbf{A})$.

\section{Bijection between partitioned locally orientable unicellular hy- permaps and permuted forests}

We proceed with the description of the bijective mapping $\Theta_{\mathbf{A}}$ between partitioned locally orientable hypermaps and permuted forests of degree $\mathbf{A}$. Let $\left(f_{3}, \pi_{1}, \pi_{2}\right)$ be a partitioned hypermap in $\mathcal{L P}(\mathbf{A})$. The first step is to define a set of white and black vertices with labeled ordered half edges such that:

- each white vertex is associated to a block of $\pi_{1}$ and each black vertex is associated to a block of $\pi_{2}$,

- the number of half edges connected to a vertex is half the cardinality of the associated block, and

- the half edges connected to the white (resp. black) vertices are labeled with the non hat (resp. hat) integers in the associated blocks so that moving clockwise around the vertices the integers are sorted in increasing order.

Then we define an ascendant/descendant structure on the vertices. A black vertex $b$ is the descendant of a white one $w$ if the maximum half edge label of $b$ belongs to the block of $\pi_{1}$ associated to $w$. Similar rules apply to define the ascendant of each white vertex except the one containing the half edge label 1.

If black vertex $b^{d}$ (resp. white vertex $w^{d}$ ) is a descendant of white vertex $w^{a}$ (resp. black vertex $b^{a}$ ) and has maximum half edge label $m$ such that $f_{3}(m)$ is the label of a half edge of $w^{a}$ (resp. $b^{a}$ ), i.e. $f_{3}\left(m^{b}\right)$ is a non hat (resp. hat) number, then we connect these two half edges to form an edge. Otherwise $f_{3}(m)$ is a hat (resp. non hat) number and we draw an arrow $(-\ldots-)$ ) between the two vertices. Note that descending edges are ordered but arrows are not.

Lemma 3.1 The above construction defines a tree structure rooted in the white vertex with half edge 1.

Proof: Let black vertices $b_{1}$ and $b_{2}$ associated to blocks $\pi_{2}^{b_{1}}$ and $\pi_{2}^{b_{2}}$ be respectively a descendant and the ascendant of white vertex $w$ associated to $\pi_{1}^{w}$. We denote by $m^{b_{1}}, m^{b_{2}}$ and $m^{w}$ their respective maximum half edge labels (hat, hat, and non hat) and assume $m^{b_{1}} \neq \widehat{n}$. As $\pi_{1}^{w}$ is stable by $f_{1}$, then $f_{1}\left(m^{b_{1}}\right)$ is a non hat number in $\pi_{1}^{w}$ not equal to 1 . It follows $m^{b_{1}}<f_{1}\left(m^{b_{1}}\right) \leq m^{w}<f_{2}\left(m^{w}\right)$. Then as $\pi_{2}^{b_{2}}$ is stable by $f_{2}$, it contains $f_{2}\left(m^{w}\right)$ and $f_{2}\left(m^{w}\right) \leq m^{b_{2}}$. Putting everything together yields $m^{b_{1}}<m^{b_{2}}$. In a similar fashion, assume white vertices $w_{1}$ and $w_{2}$ are descendant and ascendant of black vertex $b$. If we note $m^{w_{1}}, m^{w_{2}}$ and $m^{b}$ their maximum half edge labels (non hat, non hat, and hat) with $m^{b} \neq \widehat{n}$, one can show that $m^{w_{1}}<m^{w_{2}}$. Finally, as $f_{1}(\widehat{n})=1$, the black vertex with maximum half edge $\widehat{n}$ is descendant of the white vertex containing the half edge label 1 .

Example 3.2 Using the hypermap of Figure 1 we get the set of vertices and ascendant/descendant structure as described on Figure 3

Next, we proceed by linking half edges connected to the same vertex if their labels are paired by $f_{3}$ to form loops. If $i$ and $f_{3}(i)$ are the labels of a loop connected to a white (resp. black) vertex such that neither $i$ nor $f_{3}(i)$ are maximum labels (except if the vertex is the root), we assign the same label to the loop and the black (resp. white) vertex associated to the block of $\pi_{2}$ (resp. $\pi_{1}$ ) also containing $i$ and $f_{3}(i)$. 


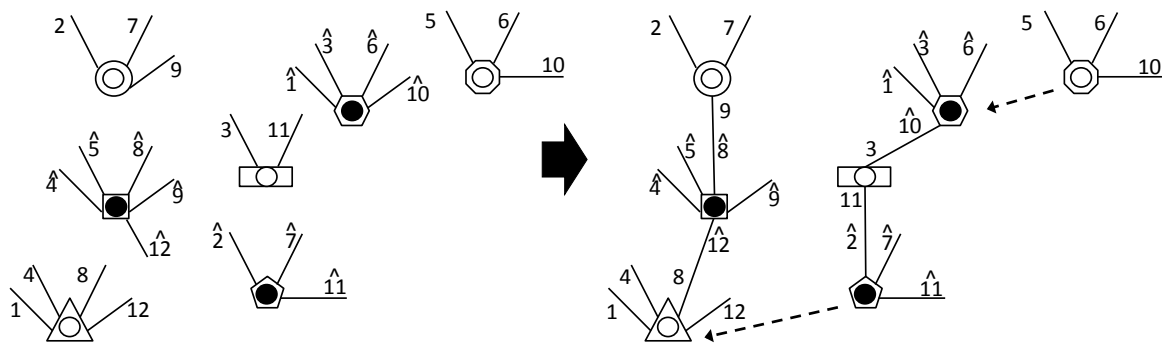

Fig. 3: Construction of the ascendant/descendant structure

As we assign at most one label from $\{\alpha, \beta, \ldots\}$ to a given vertex, several loops may share the same label.

Lemma 3.3 The number of loops connected to the vertex labeled $\alpha$ is equal to its number of incoming arrows plus the number of loops labeled $\alpha$ incident to other vertices in the forest.

Proof: The result is a direct consequence of the fact that in each block the number of hat/hat pairs is equal to the number of non hat/non hat pairs

As a final step, we define a permutation between the remaining half edges (thorns) connected to the white vertices and the one connected to the black vertices. If two remaining thorns are paired by $f_{3}$ then these two thorns are given the same label from $\{a, b, \ldots\}$. All the original integer labels are then removed. We denote by $\widetilde{F}$ the resulting forest.

Example 3.4 We continue with the hypermap from Figure 1 and perform the final steps of the construction as described on Figure 4 (Note that the geometric shapes are here for reference only, they do not play any role in the final object $F$ ).

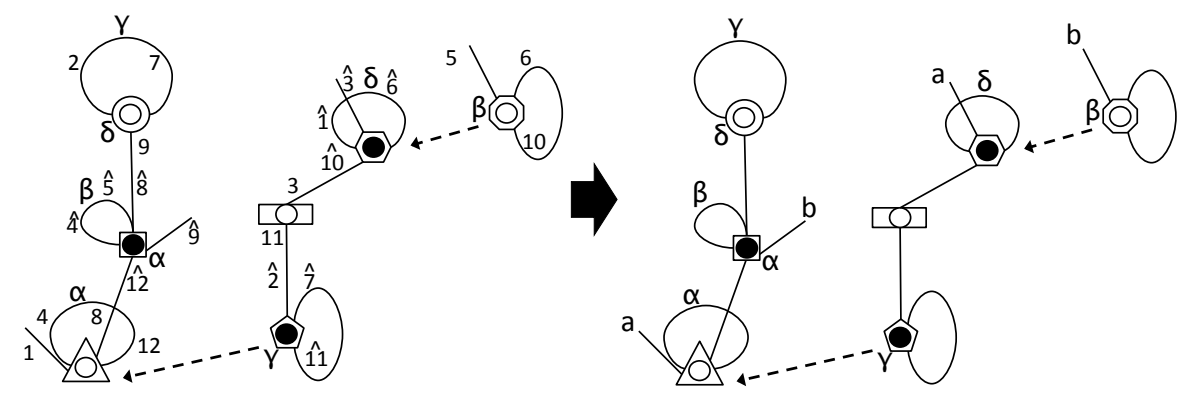

Fig. 4: Final steps of the permuted forest construction

As a direct consequence of definition $2.10, \widetilde{F}$ belongs to $\mathcal{F}(A)$. 


\section{Proof of the bijection}

We show that mapping $\Theta_{\mathbf{A}}:\left(f_{3}, \pi_{1}, \pi_{2}\right) \mapsto \widetilde{F}$ is indeed one-to-one.

\subsection{Injectivity}

We start with a forest $\widetilde{F}$ in $\mathcal{F}(\mathbf{A})$ and show that there is at most one triple $\left(f_{3}, \pi_{1}, \pi_{2}\right)$ in $\mathcal{L P}(\mathbf{A})$ such that $\Theta_{\mathbf{A}}\left(f_{3}, \pi_{1}, \pi_{2}\right)=\widetilde{F}$. The first part is to notice that within the construction in $\Theta_{\mathbf{A}}$, the original integer label of the leftmost descendant (thorn, half loop or edge) of the root vertex of the seed tree is necessarily 1 (this root is the vertex containing 1 and the labels are sorted in increasing order from left to right).

Assume we have recovered the positions of integer labels $1, \widehat{1}, 2, \widehat{2}, \ldots, i$, for some $1 \leq i \leq n-1$, non hat number. Then four cases can occur:

- $i$ is the integer label of a thorn of latin label $a$. In this case, $f_{3}(i)$ is necessarily the integer label of the thorn connected to a black vertex also labeled with $a$. But as the blocks of $\pi_{2}$ are stable by both $f_{3}$ and $f_{2}$ then $\widehat{i}=f_{2}(i)$ is the integer label of one of the descendants of the black vertex with thorn $a$. As these labels are sorted in increasing order, necessarily, $\hat{i}$ labels the leftmost descendant with no recovered integer label.

- $i$ is the integer label of a half loop of greek label $\alpha$. Then, in a similar fashion as above $\widehat{i}$ is necessarily the leftmost unrecovered integer label of the black vertex with symbolic label $\alpha$.

- $i$ is the integer label of a half loop with no symbolic label (i.e, either $i$ or $f_{3}(i)$ is the maximum label of the considered white vertex). Then, $\widehat{i}$ is necessarily the leftmost unrecovered integer label of the black vertex at the other extremity of the arrow outgoing from the white vertex containing integer label $i$.

- $i$ is the integer label of an edge. $\widehat{i}$ is necessarily the leftmost unrecovered integer label of the black vertex at the other extremity of this edge.

Finally, using similar four cases for the black vertex containing the descendant with integer label $\widehat{i}$ and the fact that blocks of $\pi_{1}$ are stable by $f_{3}$ and $f_{1}$, the thorn, half loop or edge with integer label $i+1=f_{1}(\hat{i})$ is uniquely determined as well.

We continue with the procedure described above up until we fully recover all the original labels $[n] \cup[\hat{n}]$. According to the construction of $\widetilde{F}$, the knowledge of all the integer labels uniquely determines the blocks of $\pi_{1}$ and $\pi_{2}$. The pairing $f_{3}$ is as well uniquely determined by the loops, edges and thorns with same latin labels.

Example 4.1 Assume the permuted forest $\widetilde{F}$ is the one on the right hand side of Figure 2 The steps of the reconstruction are summarized in Figure 5 . We get that the unique triple $\left(f_{3}, \pi_{1}, \pi_{2}\right)$ such that $\Theta_{\mathbf{A}}\left(f_{3}, \pi_{1}, \pi_{2}\right)=\widetilde{F}$ is:

$$
\begin{aligned}
& f_{3}=(14)(\widehat{1} \widehat{8})(29)(\widehat{2} \widehat{3})(3 \widehat{11})(\widehat{4} \widehat{10})(57)(\widehat{5} 6)(\widehat{6} 11)(\widehat{7} \widehat{9})(810) \\
& \pi_{1}=\{\{\widehat{11}, 1, \widehat{1}, 2, \widehat{2}, 3, \widehat{3}, 4, \widehat{7}, 8, \widehat{8}, 9, \widehat{9}, 10\} ;\{\widehat{4}, 5, \widehat{5}, 6, \widehat{6}, 7, \widehat{10}, 11\}\} \\
& \pi_{2}=\{\{2, \widehat{2}, 3, \widehat{3}, 5, \widehat{5}, 6, \widehat{6}, 7, \widehat{7}, 9, \widehat{9}, 11, \widehat{11}\} ;\{1, \widehat{1}, 4, \widehat{4}, 8, \widehat{8}, 10, \widehat{10}\}\}
\end{aligned}
$$

\subsection{Surjectivity}

To prove that $\Theta_{\mathbf{A}}$ is surjective, we have to show that the reconstruction procedure of the previous section always finishes with a valid output. 

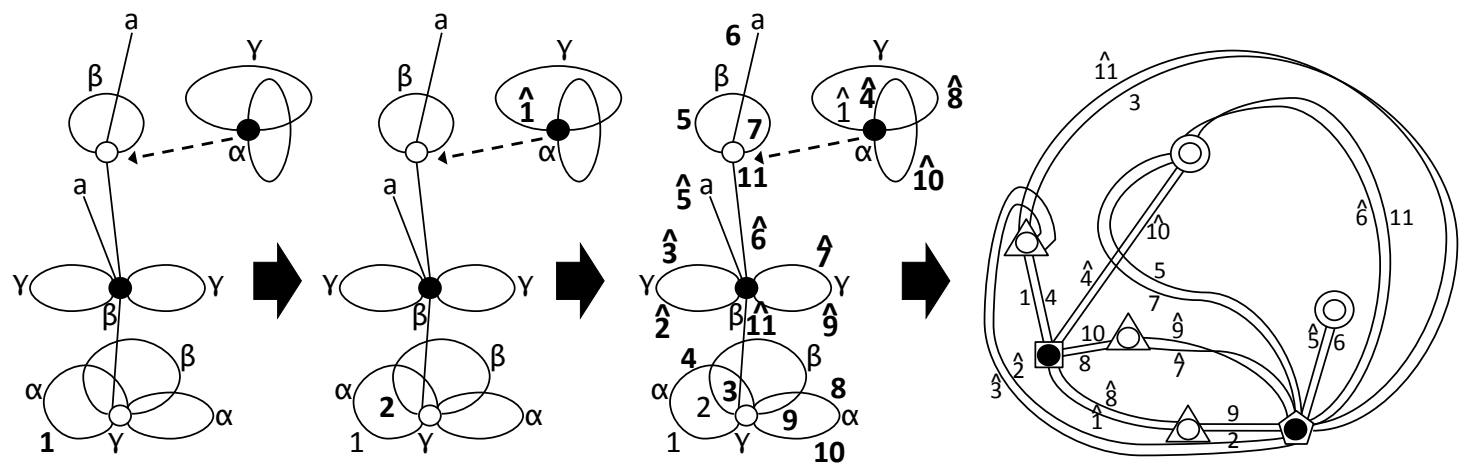

Fig. 5: Recovery of the integer labels and the partitioned map

Assume the procedure comes to an end at step $i$ before all the integer labels are recovered (where $i$ is for example non hat, the hat case having a similar proof). It means that we have already recovered all the labels of vertex $v^{i}$ identified as the one containing $\widehat{i}$ (or $\left.i+1\right)$ prior to this step. This is impossible by construction provided $v^{i}$ is not the root vertex of the seed tree since the number of times a vertex is identified for the next step is equal to its number of thorns, plus its number of edges, plus twice the number of loops that have the same greek label as $v^{i}$, plus twice the incoming arrows. Using Property (iv) of Definition 2.10. we have that the sum of the two latter numbers is twice the number of loops of $v^{i}$. As a consequence, the total number of times the recovering process goes through $v^{i}$ is exactly (and thus never more than) the degree of $v^{i}$.

If $v$ is the root vertex of the seed tree, the situation is slightly different due to the fact that we recover label 1 before we start the procedure. To ensure that the procedure does not terminate prior to its end, we need to show that the $|v|$-th time the procedure goes through the root vertex is right after all the labels of the forest have been recovered. Again, this is always true because:

- The last element of a vertex to be recovered is the label of the maximum element of the associated block. Consequently, all the elements of a vertex are recovered only when all the elements of the descending vertices (through both arrows and edges) are recovered.

- Property $(v)$ of Definition 2.10 states that the ascendant/descendant structure involving both edges and arrows is a tree rooted in $v$. As a result, the procedure goes the $v$-th time through $v$ only when all the elements of all the other vertices are recovered.

\section{On proving Theorem 1.1 using Zonal polynomials}

In the orientable case, one can use Schur symmetric functions and the irreducible characters of the symmetric group to prove the identity in Equation (7) (see [6]). This requires: (i) $\left(p_{\lambda} \rightarrow s_{\mu}\right)$ the MurnaghanNakayama rule, (ii) $\left(s_{\mu} \rightarrow m_{\nu}\right)$ finding the number of semistandard Young tableaux of hook shape $\lambda=a 1^{n-a}$ and type $\mu$ which is just $\left(\begin{array}{c}\ell(\mu)-1 \\ r\end{array}\right)$, and (iii) using inclusion exclusion. One could try to replicate this on $\Psi^{n}$ and obtain an algebraic proof of Theorem 1.1. We show the outcome after step (i)' using [5. Cor. 5.2]. Steps (ii)' and (iii)' appear quite less tractable.

(i)' $\Psi^{n}(\mathbf{x}, \mathbf{y})=\frac{\left|B_{n}\right|}{\left|K_{(n)}\right|} \sum_{a \geq b \geq 1} \frac{\varphi^{\left(a, b, 1^{n-a-b}\right)}(n)}{H_{2\left(a, b, 1^{n-a-b}\right)}} Z_{\left(a, b, 1^{n-a-b}\right)}(\mathbf{x}) Z_{\left(a, b, 1^{n-a-b}\right)}(\mathbf{y})$. 


\section{Appendix: completion of the main formula}

For Theorem 1.1 to be exact, the following additional definitions for some limit values of $\mathbf{A}$ are needed:

(i) If $q^{\prime} \neq 0$, then there is at most one given $\left(i_{0}, j_{0}, k_{0}\right)$ with $i_{0}=2\left(j_{0}+k_{0}\right)$, for which $P_{i_{0} j_{0} k_{0}}=1$ instead of 0 . In that situation, we define $\mathcal{N}(\mathbf{A})\left(\begin{array}{c}i i_{0}-1 \\ j_{0}, k_{0}, j_{0}+k_{0}\end{array}\right)^{P_{i_{0} j_{0} k_{0}}}=$

$$
\frac{j_{0}}{q^{\prime}}\left(\begin{array}{c}
i_{0} \\
j_{0}, k_{0}
\end{array}\right)\left(\frac{\delta_{p^{\prime} \neq 0}}{p^{\prime}} \sum_{i, j, k}(i-2 j-2 k) P^{\prime} \sum_{i, j, k} j Q^{\prime}+\sum_{i, j, k}(i-2 j-2 k) Q^{\prime} \frac{1+\sum_{i, j, k}(i-1-2 j-2 k) P}{n-q-2 r}\right)
$$

(ii) When $q^{\prime} \neq 0$ and $n-q-2 r=0$, which can only occur if $p=1$ (we assume $P=E_{t, u, v}$, the elementary array with $E_{t, u, v}=1$ and 0 elsewhere) the whole summand reduces to:

$$
\begin{aligned}
& \frac{1}{\mathbf{A} !}\left[\delta_{(n-2 r) p^{\prime} \neq 0} \frac{(t-2 u-2 v) \sum_{j, k, l} j Q}{(n-2 r) p^{\prime}}+\delta_{p^{\prime} \neq 0} u \frac{\sum_{i, j, k} j Q^{\prime}}{p^{\prime} q^{\prime}}+\delta_{p^{\prime}=0}\right] \\
& \times \frac{(n-2 r) ! p^{\prime} ! q^{\prime} !\left(r-p^{\prime}\right) !\left(r-q^{\prime}\right) !}{2^{2 r-p^{\prime}-q^{\prime}}}\left(\begin{array}{c}
t \\
u, v, u+v
\end{array}\right) \prod_{i, j, l}\left(\begin{array}{c}
i-1 \\
j, k, j+k
\end{array}\right)^{Q_{i j k}}\left(\begin{array}{c}
i-1 \\
j, k, j+k-1
\end{array}\right)^{\left(P^{\prime}+Q^{\prime}\right)_{i j k}}
\end{aligned}
$$

\section{Acknowledgements}

AHM thanks the Combinatorial Models Group of l'École Polytechnique for their invitation there where this work was done. EAV acknowledges the support of ERC under "ERC StG 208471 - ExploreMaps".

\section{References}

[1] O. Bernardi An analogue of the Harer-Zagier formula for unicellular maps on general surfaces, arXiv: 1011.2311

[2] I.P. Goulden and D. M. Jackson Maps in Locally orientable surfaces, the double coset algebra and zonal polynomials Can. J. Math., 48(3):569-584, 1996.

[3] I.P. Goulden and D. M. Jackson Combinatorial constructions for integrals over normally distributed random matrices Proc. Amer. Math. Soc., 123(4), 1995.

[4] I. P. Goulden and A. Nica A direct bijection for the Harer-Zagier formula. J. Comb. Theory Ser. A, 111(2):224-238, 2005.

[5] P. J. Hanlon, R. P. Stanley and J. R. Stembridge. Some Combinatorial Aspects of the Spectra of Normally Distributed Random Matrices Contemp. Math., 138:151-174, 1992.

[6] D.M. Jackson. Some combinatorial problems associated with products of conjugacy classes of the symmetric group, J. Combin. Theory Ser. A, 49:363-369 (1988).

[7] B. Lass Démonstration combinatoire de la formule de Harer-Zagier C.R. Acad. Sci. Paris 333.Série I:155-160, 2001

[8] I. G. Macdonald. Symmetric Functions and Hall Polynomials, Oxford University Press, Oxford, 2nd Edition., 1995.

[9] A. Morales and E. A. Vassilieva Bijective enumeration of bicolored maps of given vertex degree distribution DMTCS Proc. (FPSAC), AK:661-672, 2009. 